\title{
The drumstick gene acts cell-non-autonomously and triggers specification of the small intestine in the Drosophila hindgut
}

\author{
SARDER N. UDDIN ${ }^{1,2}$, MASAHIRO YANO ${ }^{1}$ and RYUTARO MURAKAMI*,1 \\ ${ }^{1}$ Department of Applied Molecular Bioscience, Graduate School of Medicine, Yamaguchi University, Japan and \\ ${ }^{2}$ Biotechnology and Genetic Engineering Discipline, Khulna University, Bangladesh
}

\begin{abstract}
An odd family gene drumstick (drm) encodes a zinc finger protein, and is necessary for the development of the small intestine, an anterior domain of the ectodermal hindgut of Drosophila melanogaster. However, mechanisms that specify the small intestine, as well as gene regulatory pathways leading to transcriptional activation of $d r m$, are still unclear. We found that $d r m$ is expressed in two different tissues abutting the anterior end of the hindgut primordium, that is, the posterior-most region of the midgut (endoderm) and basal portion of the Malpighian tubules. A small intestine marker gene, unpaired (upd), begins to be expressed at the anterior-most region of the hindgut primordium that abuts the basal portion of Malpighian tubules, and the upd-positive region expands, resulting in a short tube during stages 11-13. The small intestine develops in both of the mutant embryos, serpent (srp) and Krüppel (Kr), that lack the drm-positive midgut or Malpighian tubules, respectively, while it fails to develop in the $K r$ srp double-mutant embryos that lack both of the $d r m$-positive tissues. These results demonstrate that $d r m$ expressed in the abutting tissues cell-non-autonomously induces development of the small intestine in the hindgut primordium, probably by deploying some extracellular signaling factor. $\mathrm{drm}$ expression in the posterior gut region disappears and the small intestine fails to form in tailless (tII) mutant embryos, whereas over-expression of $t / l$ causes expansion of $\mathrm{drm}$ expression throughout the midgut, inducing a longer small intestine. These results indicate that $\mathbf{d r m}$ is activated under the control of $t / l$ and triggers development of the small intestine cell-non-autonomously through some extracellular signaling.
\end{abstract}

KEY WORDS: Drosophila, drumstick, unpaired, cell-non-autonomous, small intestine

\section{Introduction}

The digestive tract is an evolutionarily conserved and structurally simple organ composed of an epithelial tube surrounded by visceral muscles of mesodermal origin. The Drosophila gut consists of three major parts: ectodermal foregut, endodermal midgut and ectodermal hindgut. Each of these gut parts are subsequently subdivided into a number of distinct domains characterized by specific gene expression pattern (Murakami et al., 1994). These features make the Drosophila gut an attractive organ for the study of regional differentiation (Skaer, 1993; Hoch and Pankratz, 1996; Lengyel and Liu, 1998; Murakami et al., 1999; Lengyel and Iwaki, 2002). All the gut epithelia originate from anterior and posterior terminal domains of the cellular blastoderm, which form a continuous tube after invagination from both terminals. Fate decision of the both terminal domains is controlled by a gene regulatory system, the terminal system (reviewed in Nüsslein-Volhard et al., 1987; Skaer, 1993; Murakami et al., 1999). Initially, graded concentrations or activities of maternal morphogens are established, with maximum peaks at both anterior and posterior termini of the egg (Greenwood and Struhl, 1997; Martin et al., 1994; Savant-Bhonsale and Montell, 1993; Sprenger and Nüsslein-Volhard, 1992), which leads to activation of the two earliest zygotic genes tailless ( $t / l)$ and huckebein $(h k b)$ in a nested pattern (Brönner and Jäckle, 1991; Pignoni et al., 1990). brachyenteron (byn), a brachyury ortholog, is essential for specifying hindgut primordium (Kispert et al., 1994; Murakami et al., 1995; Singer et al., 1996). In the posterior terminal region of the cellular blastoderm, byn is activated by $t / l$, and repressed by a $h k b$ target gene srp, thus being restricted to a region spanning $10-15 \%$

\footnotetext{
Abbreviations used in this paper: ap, anal pads; arm, armadillo; bowl, brother of odd with entrails limited; byn, brachyenteron; ct, cut; drm, drumstick; EL, egg length; hg, hindgut/hindgut primordium; hkb, huckebein; knrl, knirps-related; Kr, Krüppel; li, large intestine; lin, lines; mg, midgut/ midgut primordium; mt, Malpighian tubules; otp, orthopedia; rec, rectum; si, small intestine; srp, serpent; st, stage; tll, tailless, upd, unpaired; wg, wingless; WT, wild-type.
}

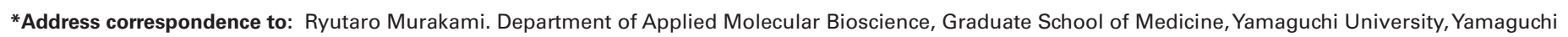
753-8512, Japan. Tel/Fax: +81.83.933.5696. e-mail: ryu@yamaguchi-u.ac.jp

ISSN: Online 1696-3547, Print 0214-6282 
egg length (EL) of the early cellular blastoderm, and the byn-positive region eventually forms the hindgut and anal pads (Reuter and Leptin, 1994; Rehorn et al., 1996; Murakami et al., 1999; Lengyel and Iwaki, 2002). During embryonic development, the hindgut primordium is subdivided into several domains characterized by specific gene expression and ultrastructure (Hoch and Pankratz, 1996; Pankratz and Hoch, 1995; Murakami and Shiotsuki, 2001; Takashima and Murakami, 2001). A major anterior domain is the small intestine, then, the large intestine follows. The large intestine is further subdivided into a dorsal and a ventral domains that are demarcated by a one-cell-wide boundary cell domain. The rectum domain arises posterior to the large intestine, and the anal pads form the posterior orifice of the gut tube. There are several studies on mechanisms of the subdivision of hindgut primordium: $\mathrm{Wg}$ signal emanated from the anal pads induces the rectum (Takashima and Murakami, 2001); boundary cells are specified by Notch signaling activated at the interface between the dorsal and ventral domains of the large intestine (Takashima et al., 2002; Iwaki et al., 2001; Fu $\beta$ and Hoch, 2002). These results imply importance of cell-to- cell interactions mediated by extracellular signal molecules in the process of subdividing hindgut into several domains. The small intestine has a couple of features similar to the rectum both in the ultrastructure and gene expression pattern: apical surface of the epithelium shows no trait of absorptive function, being covered by a thick cuticular layer; the epithelial tube is surrounded by a welldeveloped sphincter muscles (Murakami and Shiotsuki, 2001); both the small intestine and rectum express hedgehog (hh) and knirps-related (knrl) (Hoch and Pankratz, 1996; Fuß et al., 2001; Takashima and Murakami, 2001; Iwaki et al., 2001; Lengyel and Iwaki, 2002; Green et al., 2002; Johansen et al., 2003). However, while the rectum is induced by $\mathrm{Wg}$ signaling, the small intestine is independent of $\mathrm{Wg}$ (Takashima and Murakami, 2001). Odd-family genes, drumstick (drm) and bowl, as well as Wnt-pathway related gene lines (lin), are involved in the process of fate decision of the small intestine (Iwaki et al., 2001; Lengyel and Iwaki, 2002; Green et al., 2002; Johansen et al., 2003; Hatini et al., 2005). drm is essential for the development of small intestine: $d r m$ mutant embryos fail to develop small intestine, while over-expression of
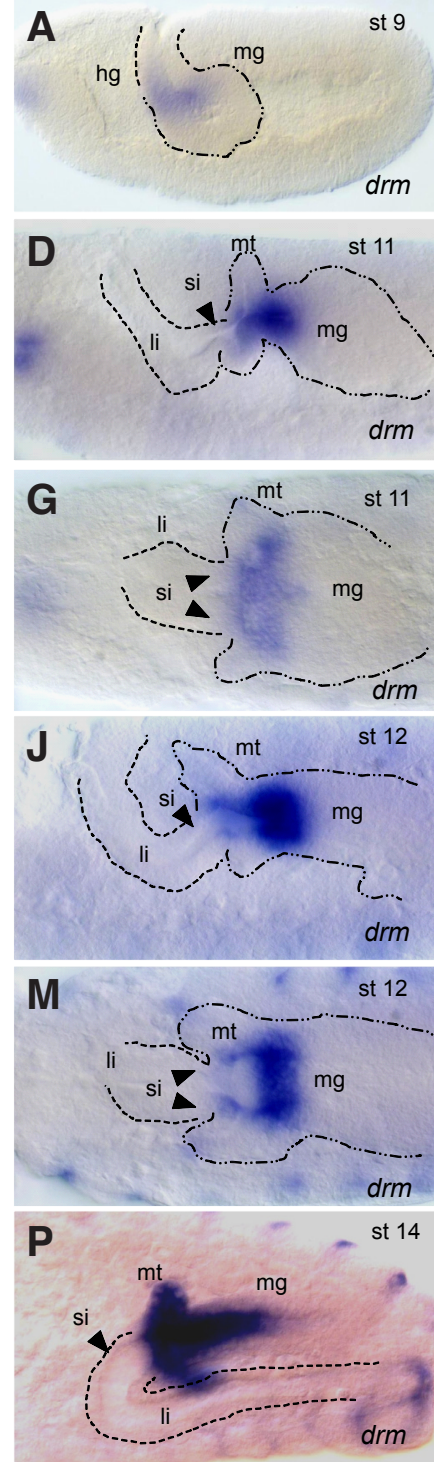
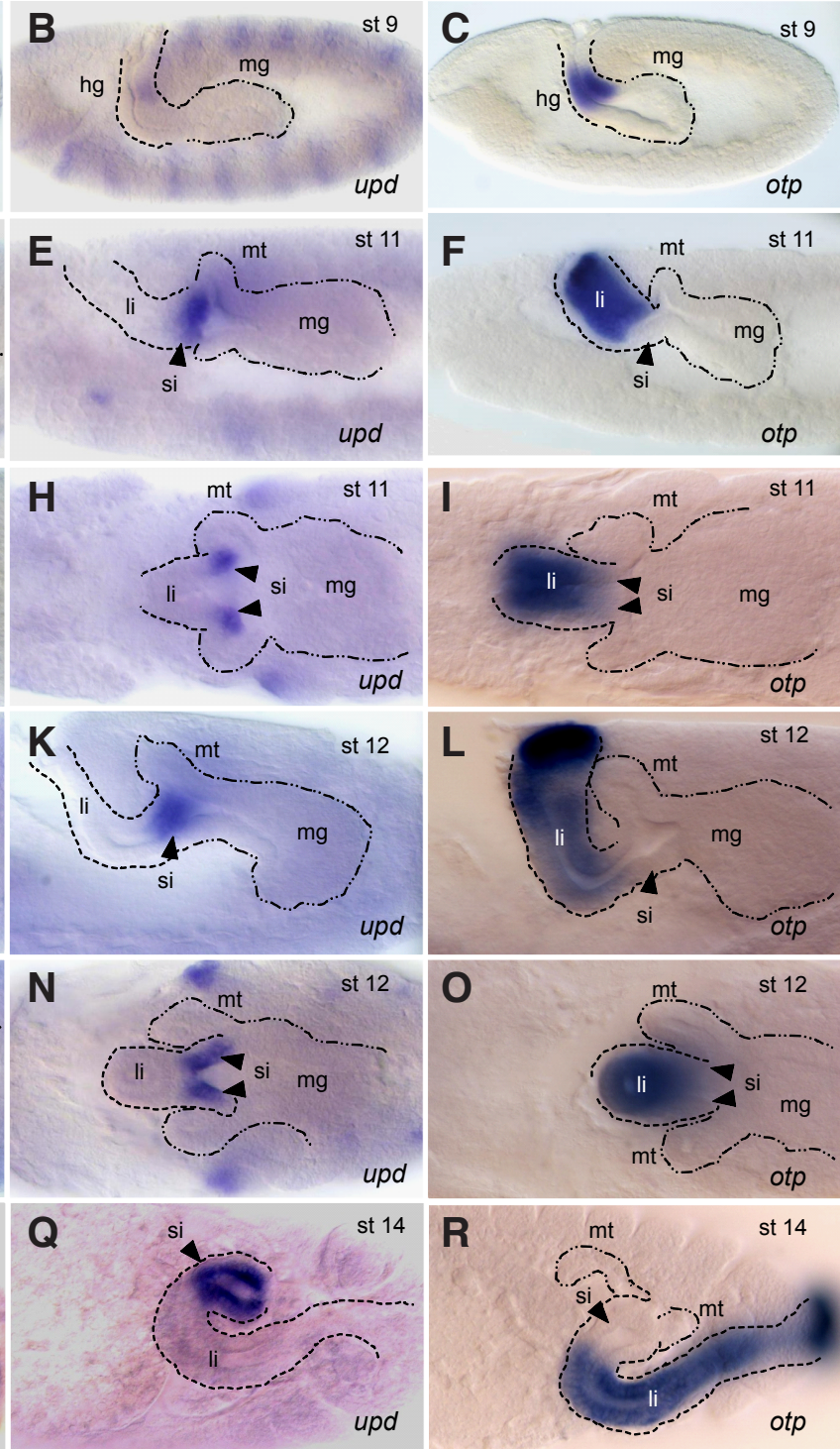

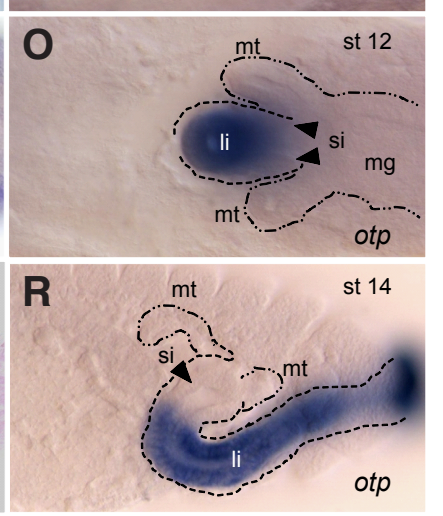

Fig. 1. Expression patterns of drm, upd and otp mRNAs during development of the small intestine in wild-type embryos. In all panels, anterior of the embryos is to the left. Embryonic stages and detected $m R N A s$ are indicated at the top right and bottom right of the panels, respectively. (AC) Stage 9 embryos, lateral views: (A) Weak and diffuse drm signal in the hindgut (hg) and midgut primordium (mg) is detected., (B) No upd signal appears yet., (C) otp begins to be expressed in the hindgut primordium. (D-I) Stage 11 embryos: (D-F) Lateral views, and (G-I) dorsal views., (D,G) drm expression becomes distinct in the posterior-most region of midgut and basal portion of the Malpighian tubules, not in the small intestine domain (si, arrowheads)., (E,H) upd is expressed as bilateral spots representing developing small intestine domain (arrowheads)., (F,I) otp signal is disappearing in the small intestine (arrowheads). (J-O) Stage 12 embryos: (JL) Lateral views and (M-O) dorsal views., (J,M) drm expression becomes restricted to the posterior-most region of midgut and basal portion of the Malpighian tubules., $(\mathbf{K}, \mathbf{N})$ upd positive region expanded posteriorly, partially becoming tubular., $(\mathbf{L}, \mathbf{O})$ The small intestine (arrowheads) is negative for otp signal. (P-R) Stage 14 embryos, lateral views: (P) drm is exclusively expressed in the posterior-most region of midgut and basal portion of the Malpighian tubules., (Q) upd is expressed exclusively in the small intestine., (R) otp is expressed in the large intestine. Abbreviations: hg, hindgut/ hindgut primordium; li, large intestine; mg, midgut/midgut primordium; mt, Malpighian tubules; si, small intestine. 
$d r m$ transforms most of the hindgut primordium into small intestine. However, both upstream and downstream regulatory pathway of $d r m$ is still unclear. In the present study, we show that $d r m$ is activated under the control of $t I l$, and, acts cell-non-autonomously in the process of specifying small intestine, probably deploying some extracellular signaling factor.

\section{Results}

drumstick (drm) is expressed in the posterior-most region of the midgut and basal portion of the Malpighian tubules, but not in the small intestine during late stages

Weak and rather diffuse signal of $d r m$ is detectable in the invaginating proctodeum as early as stage 7 . During stage 9 and 10 , $d r m$ expression is still weak and diffuse, and the positive region apparently includes the future hindgut and posterior-most region of the midgut (endoderm) (Fig. 1A). unpaired (upd), a marker gene of the small intestine, is not expressed in the hindgut region at these stages (Fig. 1B). On the other hand orthopedia (otp), a homeobox gene specifically expressed in the large intestine, is first expressed in the hindgut primordium at stage 9 (Fig. 1C). At stage 11, the expression pattern of $d r m$ becomes distinct, with $d r m$-positive area being restricted to the posterior-most region of the midgut and basal portion of the buds of Malpighian tubules (Figs. $1 \mathrm{D}, \mathrm{G}$ ). When viewed dorsally, upd signal first appears at this stage as bilateral spots abutting the buds of Malpighian tubules (Fig. $1 \mathrm{H})$. Concomitantly, otp signal in the anterior-most region of the hindgut primordium becomes very weak (arrowheads in Figs. $1 \mathrm{~F}, \mathrm{I})$. During stage 12, the upd-positive region expands posteriorly and medially, becoming tubular (Figs. $1 \mathrm{~K}, \mathrm{~N}$ ), and border between the upd-positive small intestine and otp-positive large intestine becomes clear (Figs. $1 \mathrm{~K}, \mathrm{~L}, \mathrm{~N}, \mathrm{O}$ ). During late stage 12 and 13, the upd-positive domain eventually forms a complete tube. At stage 14, all the domains in and around the small intestine can be recognizable by specific gene expression: $d r m$ for the posterior-most region of midgut and basal portion of the Malpighian tubules (Fig. 1P); upd for the small intestine (Fig. 1Q); otp for the large intestine (Fig. $1 \mathrm{R})$. These observations indicate that $\mathrm{drm}$ is expressed in tissues abutting anterior end of the hindgut primordium after stage 11.

\section{drumstick mutant embryos fail to form the small intestine while adjacent tissues remain largely intact}

Above results suggest that $d r m$ works cell-non-autonomously in the development of small intestine. We examined effects of $d r m$ mutation on the small intestine and abutting tissues by use of region-specific probes to know what tissues are affected by the $d r m$ mutation.

$k n r l$ and upd have been used as marker genes of the small intestine (Figs. 2 A,C) (Harrison et al., 1998; Fu $\beta$ et al., 2001; Iwaki et al., 2001; Green et al., 2002; Johansen et al., 2003). In $d r m$ mutant embryos, hindgut is short, and there is no expression of $\mathrm{knrl}$ and upd in the anterior region of the hindgut (asterisks in Figs. 2 B,D), being consistent with the previous reports (Green et al., 2002; Johansen et al., 2003). To examine effects of $d r m$ mutation on the tissues abutting the small intestine, in situ hybridization was carried out using the following probes: GATAe probe for the endoderm/Malpighian tubules; otp probe for the large intestine; cut probe for the Malpighian tubules. In wild-type embryos, small intestine is clearly recognizable as a short tube negative for GATAe
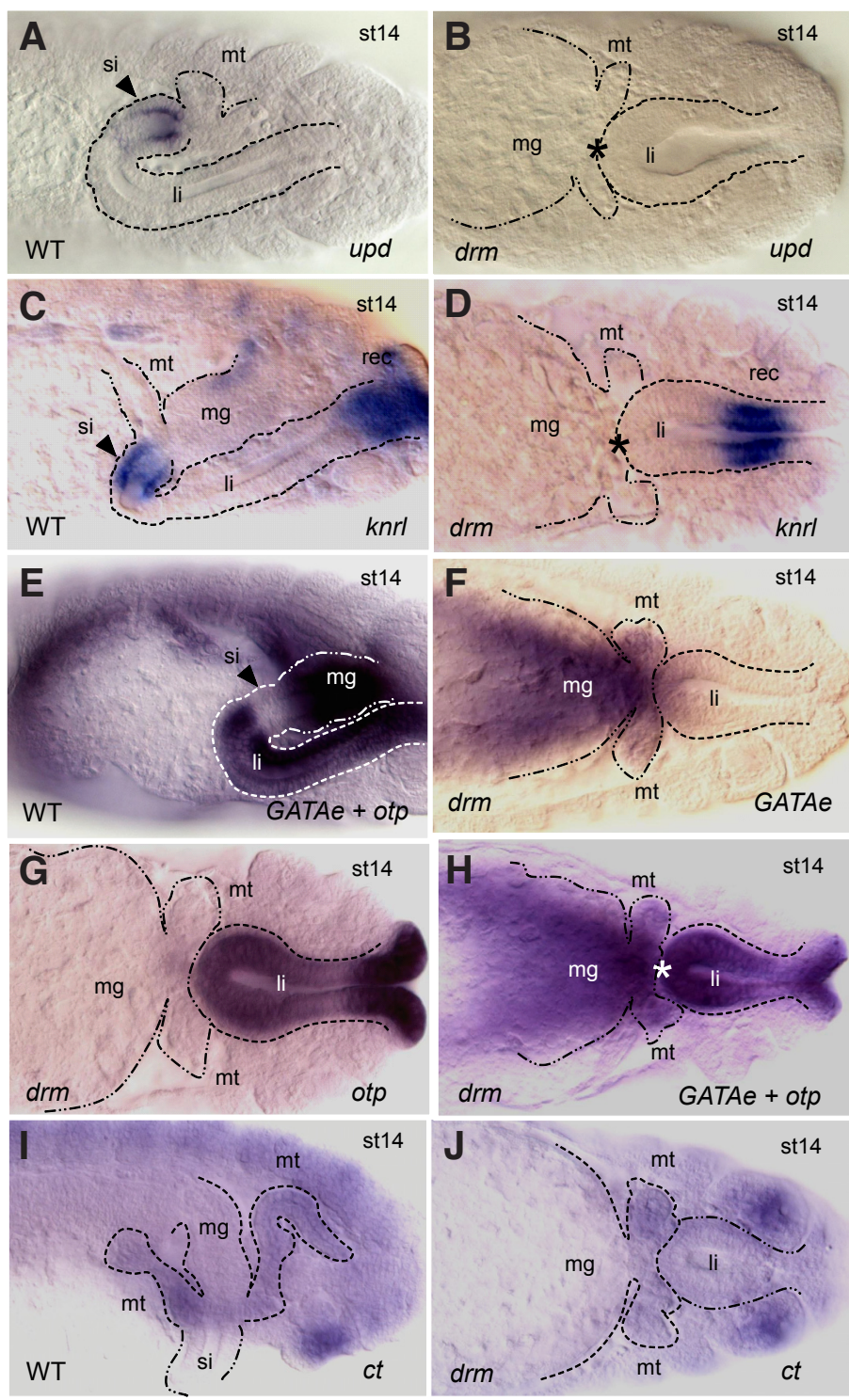

Fig. 2. drm mutant embryos fail to form small intestine while adjacent tissues remain largely intact. In all panels, anterior of the embryos is to the left. Embryonic stages and detected mRNAs are indicated at the top right and bottom right of the panels, respectively, and mutant names

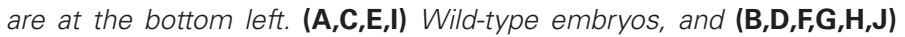
drm mutant embryos: (A) upd is expressed in the small intestine of the wild-type embryo. (B) upd expression disappears (asterisk) in the drm mutant embryo. (C) $\mathrm{knrl}$ is expressed in the small intestine and rectum in the wild-type embryo. (D) In drm mutant, the small intestine does not form (asterisk), and $\mathrm{knrl}$ expression remains only in the rectum. (E) GATAe-otp double-staining of the wild-type embryo. The small intestine is recognized as a domain negative for both signals (arrowhead). (F) In drm mutant, GATAe is expressed in the midgut and the buds of Malpighian tubules. (G) In drm mutant, otp is normally expressed in the large intestine. (H) GATAe-otp double-staining of the drm mutant embryo. There is no gap between GATAe and otp expression domain, indicating that the small intestine is completely missing (asterisk). (I) Expression of cut (ct) in wild-type embryo. Malpighian tubules are positive for ct. (J) In the drm mutant embryo, ct is still expressed weakly in the Malpighian tubules. Abbreviations: li, large intestine; mg, midgut; mt, Malpighian tubules; rec, rectum; si, small intestine. 

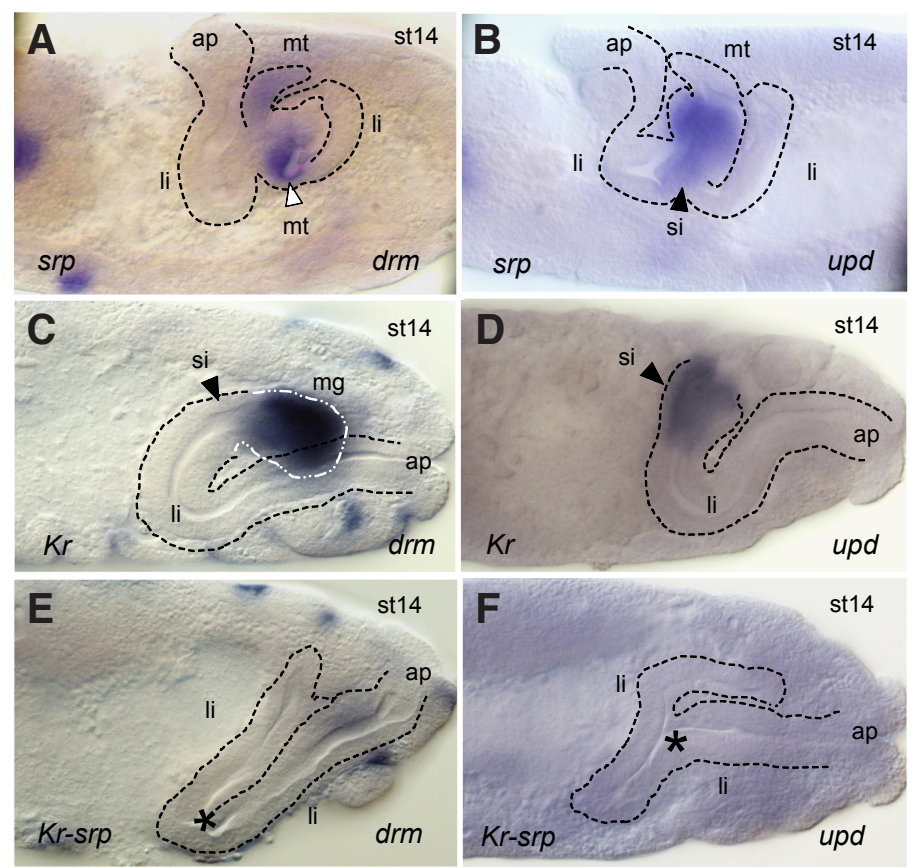

and otp probes (Fig. 2E). In drm mutant embryos, expression of both marker genes remains largely intact (Figs. 2 F,G). It should be noted that buds of the Malpighian tubules form and express GATAe in the drm embryos (Fig. 2F). However, small intestine that is negative for both marker genes does not form, resulting in a continuous staining region positive for GATAe-otp mixed probes at the midgut-hindgut junction (asterisk in Fig. $1 \mathrm{H}$ ). These results demonstrate that in $\mathrm{drm}$ mutant embryos, small intestine does not form, and also suggest that adjacent tissues that express $d r m$ in normal embryos remain largely intact. Actually, buds of Malpighian tubules express cut (ct), a marker genes of the tissue, although the signals are weaker than those in wild-type embryos (Figs. $2 \mathrm{I}, \mathrm{J}$ ). Regarding the posterior-most domain of the midgut, its macroscopic morphology in later embryonic stages looks slightly abnormal, with rather short convoluted portion in the posterior-most region, suggesting $\mathrm{drm}$ mutation affects development of this region. Although marker genes specific for this region in differentiated midgut are not available, $w g$ is known

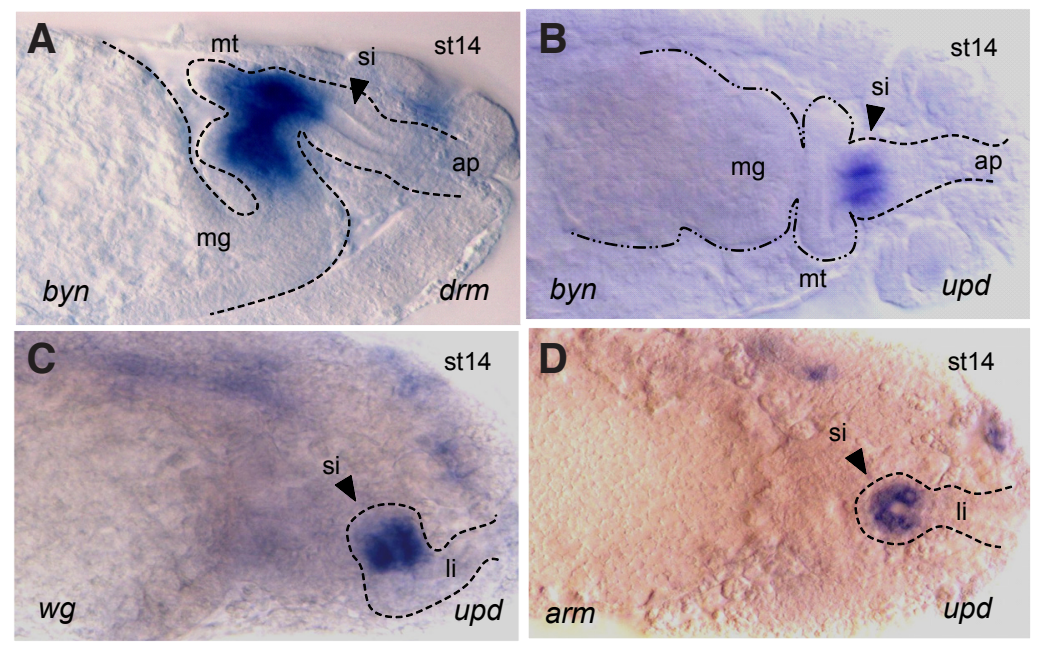

Fig. 3. Development of the small intestine in the mutants that lack drm-positive tissues. In all panels, anterior of the embryos is to the left. Mutant names are indicated at the bottom left of the panels. Embryonic stages and detected $m R N A$ s are at the top right and bottom right, respectively. $(\mathbf{A}, \mathbf{B})$ srp mutant embryos, in which prospective midgut transforms into large intestine. (A) drm is expressed in rudimentary Malpighian tubules ( $m$ t, arrowhead) between innate and supernumerary large intestine. (B) upd-positive small intestine tissue appears (arrow head), being intermingled with rudimentary Malpighian tubules. (C,D) $\mathrm{Kr}$ mutant embryos, in which Malpighian tubules do not develop. (C) drm is expressed in the posteriormost region of the midgut. (D) upd-positive small intestine develops. (E,F) Kr srp double-mutant embryos. Asterisks indicate approximate position of the boundary between innate and supernumerary hindgut. (E) drm expression disappears in the $\mathrm{Kr}$ srp double-mutant embryo. (F) upd expression also disappears in the $\mathrm{Kr}$ srp double-mutant embryo, indicating that the small intestine does not develop. Abbreviations: ap, anal pads; li, large intestine; mg, midgut; mt, Malpighian tubules; si, small intestine.

to be expressed in the midgut-hindgut junctional region (mainly on the midgut side). We found that $w g$ expression in this region disappears in drm mutant embryos (data not shown). This result implies a possibility that $\mathrm{drm}$ activity is mediated by $\mathrm{Wg}$ signaling, which we consider afterward (see Fig. 4).

\section{Development of the small intestine in mutations that affect drumstick-positive adjacent tissues}

Above results strongly suggest that $\mathrm{drm}$ induces small intestine cell-non-autonomously. $\mathrm{drm}$ is expressed in two different tissues, the posterior-most region of midgut and basal portion of the Malpighian tubules. Either one or both of the two drmpositive tissues are thought to be responsible for inducing small intestine. To address this issue, we examined mutants that fail to form each of the drm-positive tissues, the midgut epithelium and Malpighian tubules. srp is a key gene that specifies developmental fate of the midgut (endoderm). In srp mutant embryos, prospective anterior and posterior midgut domains transform into a portion of the ectodermal foregut and hindgut, respectively. Thus, prospective posterior midgut in srp embryos transforms into a supernumerary hindgut (mostly large intestine) (Abel et al., 1993; Rehorn et al., 1996; Reuter, 1994). In srp mutant embryos, drm expression is observed in a deformed epithelial tissue mass between the innate and supernumerary large intestine (Fig. 3A), which is assumed to represent rudimental Malpighian tubules. In the same region, strong upd expression is also detected (Fig. 3B), which is thought to represent rudimental tissues of the small intestine intermingled with rudimental Malpighian tubules. These results demonstrate that the

Fig. 4. byn andWg signaling is not necessary for the development of small intestine. In all panels, anterior of the embryos is to the left. Mutant names are indicated at the bottom left of the panels. Embryonic stages and detected mRNAs are indicated at the top right and bottom right, respectively. $(\mathbf{A}, \mathbf{B})$ byn mutant embryos. (A) drm is expressed in the posterior-most region of midgut and Malpighinan tubules in the byn mutant embryo. (B) upd-positive small intestine also developed (arrow head). (C) wg mutant embryo. The small intestine is recognized as upd-positive domain. (D) arm mutant embryo. The small intestine is recognized as upd-positive domain. Abbreviations: ap, anal pads; li, large intestine; mg, midgut; mt, Malpighian tubules; si, small intestine. 


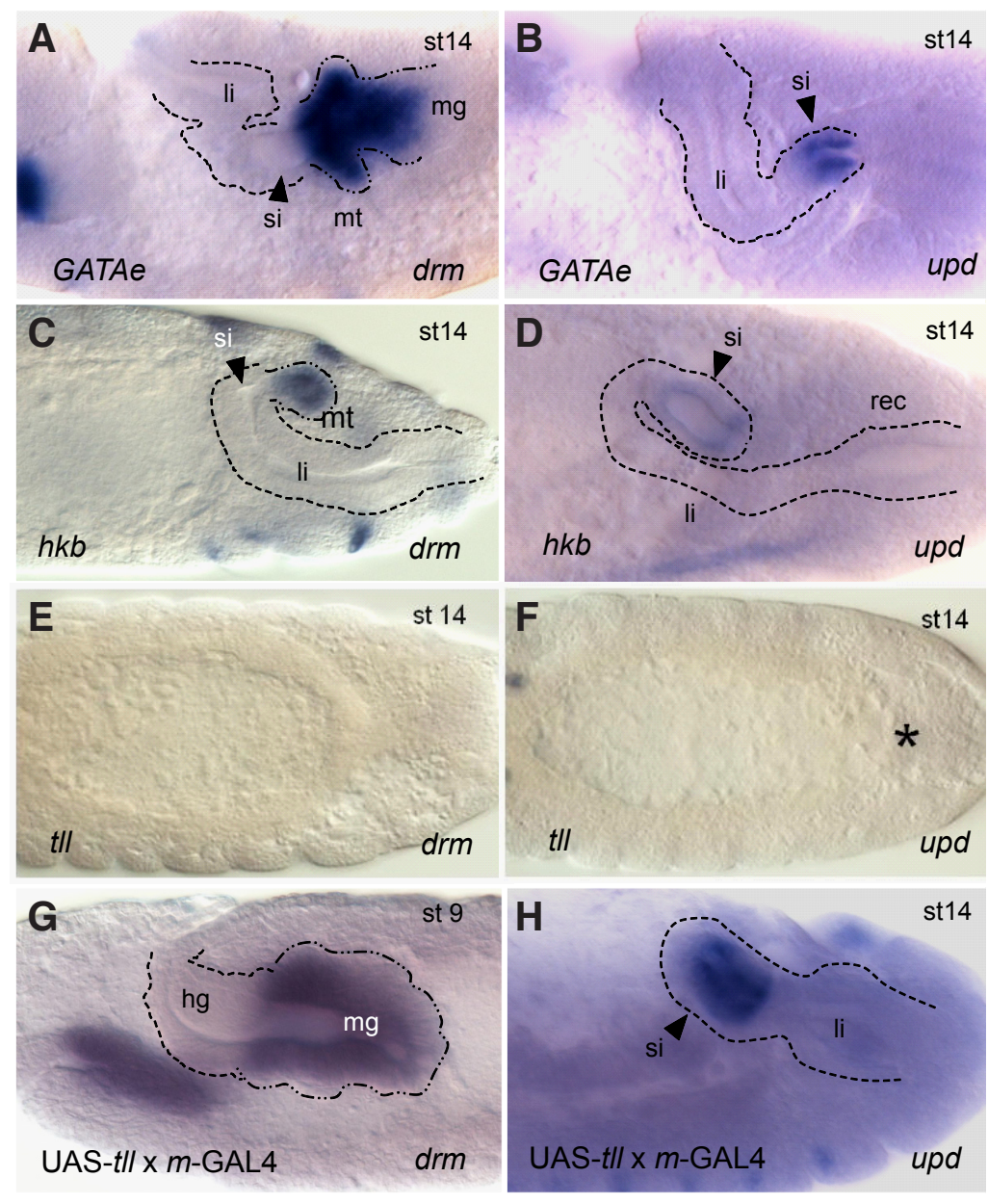

Fig. 5. Tailless (tII) is necessary for the drm expression. In all panels, anterior of the embryos is to the left. Mutant names are indicated at the bottom left of the panels. Embryonic stages and detected mRNAs are indicated at the top right and bottom, respectively. (A,B) GATAe deficient embryos. (A) drm is expressed normally at the posterior-most region of midgut and Malpighian tubules. (B) upd-positive small intestine develops normally (arrowhead). (C,D) hkb mutant embryos. (C) drm is expressed in basal portion of the Malpighian tubules. (D) upd-positive small intestine develops normally (arrowhead). (E,F) tll mutant embryos. (E) drm expression is missing in the tll mutant embryo. (F) Expression of upd is abolished in the tll mutant embryo (asterisks). (G,H) Over-expression of tll with maternal-GAL4, (G) drm expression is ectopically induced throughout the midgut. (H) Expression domain of upd markedly expands (compare with that in Fig. 2A). Abbreviations: li, large intestine; mg, midgut; mt, Malpighian tubules; rec, rectum; si, small intestine.

al., 1994; Murakami et al., 1995; Singer et al., 1996). We found that the strongest byn allele (byn $n^{\text {apro }}$ ) does not affect drm expression in the adjacent midgut and Malpighian tubules (Fig. 4A), and, unexpectedly, residual short epithelial tube expresses the small intestine marker upd (Fig. 4B) and knrl (data not shown). We also confirmed that a large intestine marker otp is abolished completely in byn embryos as was reported previously (data not shown). These results demonstrate that the small intestine can develop in the absence of byn activity, and it does not need adjacent large intestine. Next, we tried to identify extracellular signaling that mediates $d r m$ activity. Various Wnt family genes including wingless $(\mathrm{wg}$ ) are expressed at the junctional region between midgut and hindgut, and we found that wg expression in the midgut-hindgut junction disappears in $d r m$ mutants (data not shown). However, the small intestine develops in wg mutant embryos (Fig. 4C). The small intestine also develops in armadillo (arm) mutant embryos (Fig. 4D). Moreover, forced-expression of active form of arm (armS10) does not affect development of the small intestine while entire large intestine transforms into rectum (data not shown). Thus, extracellular signaling factor that is supposed to mediate $d r m$ activity remains unknown. We then examined candidates of upstream genes that regulate $d r m$ expression.

TABLE 1 two drm-positive tissues, the midgut or Malpighian tubules. In other words, either one of the $d r m$-positive tissues may be sufficient for inducing small intestine. Thus, we generate $\mathrm{Kr} s \mathrm{sp}$ double-mutant embryos to eliminate both $\mathrm{drm}$-positive tissues. In the double-mutant embryos, drm expression in the posterior gut tissues is completely abolished (Fig. 3E), and, upd expression is not detected (Fig. 3F). These results are summarized in Table 1 , and they unequivocally demonstrate that it is the $d r m$-positive tissues abutting hindgut primordium that induces small intestine, and that the action of $d r m$ is cell-non-autonomous.

\section{The small intestine is specified independently of brachyen- teron (byn) and wingless (wg) signaling}

byn is a T-box gene orthologous to the vertebrate brachyury gene, and it has been recognized as a master gene in the hindgut development. In byn mutant embryos, hindgut does not develop, or, only a very short epithelial tube remains (Kispert et

\section{TABLE}

\section{SUMMARY OF THE GUT PHENOTYPES OF THE MUTATIONS: $S R P, K R, K R$ SRP, DRM}

\begin{tabular}{|c|c|c|}
\hline Mutation & Tissue formation & $d r m$ expression in each tissue \\
\hline WT & $\begin{array}{l}\mathrm{Mg} \\
\mathrm{mt} \\
\mathbf{s i}\end{array}$ & $\begin{array}{l}+ \\
+\end{array}$ \\
\hline srp & $\begin{array}{l}\text { no mg } \\
\mathrm{mt} \\
\text { si }\end{array}$ & - \\
\hline$K r$ & $\begin{array}{l}\mathrm{mg} \\
\text { no } \mathrm{mt} \\
\text { si }\end{array}$ & $\begin{array}{l}+ \\
-\end{array}$ \\
\hline Kr srp & $\begin{array}{l}\text { no } \mathrm{mg} \\
\text { no } \mathrm{mt} \\
\text { no si }\end{array}$ & - \\
\hline drm & $\begin{array}{l}\mathrm{mg} \text { (affected?) } \\
\mathrm{mt} \\
\text { no si }\end{array}$ & - \\
\hline
\end{tabular}

Abbreviations: mg, midgut; mt, Malpighian tubules, si, small intestine 


\section{drumstick is activated under the control of tailless (tII)}

$d r m$-positive midgut region, as well as the ectodermal small intestine, arises from a posterior region of the cellular blastoderm. In order to find gene regulatory pathway leading to the drmexpression in posterior gut tube, we examined mutants of the genes that are expressed in posterior terminal region of the cellular blastoderm stage. GATAe is a GATA factor gene that is required for activating a large part of the genes expressed in the differentiated midgut epithelium (Murakami et al., 2005; Okumura et al., 2005). Embryos deficient for GATAe, however, were found to express $d r m$ in the posterior-most region of the midgut and in the basal portion of the Malpighian tubules (Fig. 5A), and the small intestine develops normally, as is proven with the upd expression (Fig. 5B). Mutation of a gap gene $h k b$, one of the earliest zygotic genes activated in the future endodermal region, was also examined. $d r m$ expression remains in the anterior end of the proctodeal invagination which corresponds to the region of Malpighian tubules (Fig. 5C), and the small intestine develops normally (Fig. 5D). $t / l$ is another gap gene expressed in the posterior blastodermal region, and its mutation is known to affects wide blastodermal region including future hindgut, Malpighian tubules as well as a posterior portion of the midgut (Brönner and Jäckle, 1991; Pignoni et al., 1990). In t/l mutant embryos, $d r m$ expression is not detected in any stages in the posterior gut region (Fig. 5E). At the same time, upd expression is not detected in the posterior gut region (Fig. 5F). The disappearance of $d r m$ expression in the posterior gut region may be indirect outcome of the drastic $t / l$ phenotype, since most of the posterior gut regions fail to form in $t / /$ mutants (Skaer, 1993). However, when $t / l$ is over-expressed by use of maternal-GAL4 (rom) driver, drm is ectopically induced throughout the endoderm (Fig. 5G), and, at the same time, the small intestine becomes larger (Fig. $5 \mathrm{H}$ ). These results strongly suggest some essential role of $t / l$ in the activation of $d r m$ as well as in the induction of small intestine.

\section{Discussion}

\section{drumstick is required cell-non-autonomously for specifying small intestine}

An odd family gene $d r m$ is essential for the development of small intestine of the Drosophila ectodermal hindgut. drm mutant exhibits very short hindgut, and fails to form the small intestine, an anterior domain of the ectodermal hindgut. In contrast, the small intestine domain expands when $\mathrm{drm}$ is forced-expressed throughout the hindgut (Iwaki et al., 2001; Lengyel and Iwaki, 2002; Green et al., 2002; Johansen et al., 2003). From extensive genetic analyses in their studies, Johansen et al., (2003) proposed a gene regulatory model for the specification of the small intestine. In their model, $d r m$ that is expressed in the anterior of the hindgut primordium induces small intestine by suppressing the activity of widely-expressed transcription factor Lin. However, as is described in the RESULTS, we found that distinct drm expression was observed exclusively in the posterior-most region of the midgut and basal portion of the Malpighian tubules from stage 11 onward, but, not in the hindgut primordium itself. $K r$ mutant embryos lack the Malpighian tubules while the midgut remains intact. On the other hand, srp embryos lack the midgut while Malpighian tubules remain intact. Both of the single mutations do not affect initial development of the small intestine. However, $\mathrm{Kr}$ srp double-mutant embryos, which lack both of the drm-positive tissues, fail to form the small intestine. These results unequivocally demonstrate that $d r m$ works in the posterior-most region of the midgut and basal portion of the Malpighian tubules, and induces the small intestine in the adjacent hindgut primordium cell-non-autonomously. In this process, Drm is assumed to activate some extracellular signaling factor, and the latter acts on the hindgut primordium and triggers developmental program of the small intestine. Canonical Wnt signaling is not essential for this process, since arm mutant, which lacks common transcription factor of the Wnt signaling pathway, does not affect initial development of the small intestine. Furthermore, when constitutive active form of arm (armS10) was forced-expressed throughout the hindgut primordium, no marked change was observed in the small intestine (data not shown). Thus, signaling factor deployed in the specification of small intestine remains still unknown.

The $d r m$-positive region of the midgut corresponds to the domain $\mathrm{m} 13$ that was recognized by its characteristic gene expression pattern in larva (Murakami et al., 1994). This region, as is the case of other posterior midgut regions, gives rise to a reabsorptive tissue in larva (Bodensein, 1950). It remains to be elucidated whether this region possesses characteristic cytological features. In embryos, the region, together with the Malpighian tubules, may act as a signaling center that affects adjacent hindgut primordium under the control of Drm. In addition to the induction of small intestine, the $d r m$-positive regions may possibly play some role in the differentiation of the posterior midgut, since our preliminary study revealed that $d r m$ mutation abolishes expression of a marker gene specific to a posterior region of the midgut.

upd-positive small intestine cells first appeared at stage 11 as bilateral spots abutting the buds of Malpighian tubules, and soon formed short tube during stage 12-13. There have been reports that prospective region of the small intestine stops cell division and DNA replication around stage 11 (Iwaki et al., 2001; Fuß et al., 2001). In fact, approximate numbers of upd-positive cells were 16 per one side at stage 11, and increased only to 20-22 at stage 13-14 (data not shown). Thus, morphological change of the small intestine during stage 11-13 may be brought about mainly by cell-rearrangement and cell-shape changes of the upd-positive cells.

\section{Upstream regulatory pathway of drumstick activation in the posterior gut region}

In order to elucidate upstream gene regulatory pathway of the drm expression, we examined several mutants that show defects in the posterior gut region. Among the mutants investigated, $t / l$ was the only mutation that abolished $d r m$ expression. $t / l$ is one of the earliest zygotic genes that are expressed in both anterior and posterior terminal regions of the cellular blastoderm (Brönner and Jäckle, 1991; Pignoni et al., 1990). Tissues including posterior gut regions around midgut-hindgut junction are impaired in tIl mutant embryos. Thus, the above result that $d r m$ expression in the posterior gut region disappeared in $t / l$ mutant embryos does not necessarily mean that $d r m$ is activated under the control of tIl. Rather, it could be an indirect effect resulting from strong defects caused in the prospective posterior gut region during early stages of development. However, forced-expression of $t / l$ throughout the embryo was found to cause ectopic expression of $d r m$ throughout the developing midgut, which strongly suggests that $d r m$ is activated under the control of $t I l$. In normal development, 


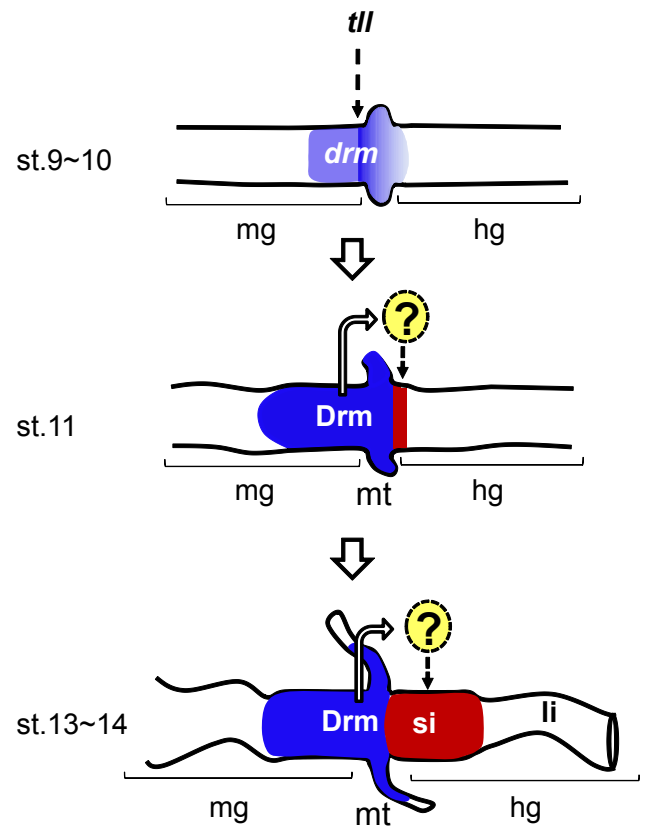

$d r m$-positive tissues

(?) unknown factor

small intestine

Fig. 6. A model of gene regulatory pathway for specification of the small intestine. drm is activated under the control of $\mathrm{tll}$, being expressed in the posterior-most region of midgut as well as in the basal portion of Malpighian tubules. Subsequently, Drm protein activates unknown extracellular signaling factor and the latter acts on the hindgut primordium and triggers specification of the small intestine at stage 11. Abbreviations: hg, hindgut; li, large intestine; mg, midgut; mt, Malpighian tubules; si, small intestine. The color codes are indicated at the figure bottom.

$d r m$ expression is restricted only to the posterior-most region of midgut and basal portion of the Malpighian tubules from stage 11 onward. Some unknown factors, in conjunction with $t / l$, may be involved in defining the spatial expression pattern of $\mathrm{drm}$ in these regions. A model of gene regulatory pathway leading to the specification of small intestine, which is deduced from the present and previous studies, is presented in Fig. 6.

\section{Signaling pathway of the development of small intestine is still puzzling}

In the present study, we revealed a few essential steps in the pathway of small intestine development. However, mechanisms of the specification of small intestine are still rather ambiguous and puzzling. In the model of Johansen et al., (2003), drm suppresses activity of the ubiquitously-expressed transcription factor Lin, and, Lin suppresses activity of another odd-family protein Bowl in the absence of $d r m$ activity. Thus, $d r m$ activity eventually leads to the activation of Bowl, and the latter is essential for determining tissue identity of the small intestine. However, there is no direct evidence that demonstrates specific roles of Bowl in this process. All these circumstantial knowledges suggest that there may be some unknown factor that defines identity of the small intestine. Thus, most important issues to be solved is: extracellular signaling factor generated under the control of $\mathrm{drm}$, and, gene that determines tissue identity of the small intestine.

\section{Materials and Methods}

\section{Fly strains}

The following fly stocks are used: Oregon $\mathrm{R}$ as wild type for the analyzing normal development, wgPY40 (hypomorphic mutant, Murakami et al., 1994); byn apro (strongest allele, Murakami et al., 1995); srp $^{2}$ (amorphic allele; Reuter, 1994); $h k b^{A}$ (null allele); $D f(3 R) s b d 45$ (deletion of GATAe locus); arm $4 m$ (null mutant of arm); $D f(2 L) d r m P 1$ (chromosomal deletion of $d r m$ locus); $K r^{2}$ (amorphic mutant); $t / I^{G}$ (null allele); $K r^{2}$ srpdouble mutant was generated by conventional crossings.

The following GAL4 and UAS strains were used for misexpression experiments: byn-GAL4 (for misexpression in the hindgut primordium); 48Y-GAL4 (for misexpression in the midgut); maternal-GAL4 (ubiquitous); UAS-tll;UAS-wg;UAS-drm; UAS-armS10. All these strains are provided by DGRC, Kyoto Stock Center and Bloomington Stock Center, if not mentioned specifically. The flies were raised at $24^{\circ} \mathrm{C}$ for collection of the embryos.

\section{In situ hybridization}

The following cDNAs or EST clones were used as template to synthesis DIG-labeled RNA probes: upd cDNA (Harrison et al., 1998, EST clone AT23111, also known as outstretched, os -flybase); drm cDNA (Green et al., 2002, EST clone LD26791); knrl cDNA (Chen et al., 1998); otp cDNA (Simeone et al., 1994); GATAe cDNA (EST clone LD08432); ct cDNA (EST clone RE08418); wg cDNA (Baker, 1987). The whole mount in situ hybridization was carried out as described by Tautz and Pfeifle, (1989). Staging of the embryos were done according to Campos-Ortega and Hartenstein, (1985).

\section{Acknowledgements}

We thank DGRC Kyoto Stock Center and Bloomington Stock Center for providing fly strains used in this study. This study is supported in part by Grants-in-Aid from Japan Society for the Promotion of Science (JSPS) (22570205) to R. M., and S. N. U. is supported by Japanese Government Scholarship.

\section{References}

ABEL, T., MICHELSON, A.M. and MANIATIS, T.A. (1993). Drosophila GATA family member that binds to $A D H$ regulatory sequences is expressed in the developing fat body. Development 119: 623-633.

BAKER, N. (1987). Molecular cloning of sequences from wingless, a segment polarity gene in Drosophila, the spatial distribution of a transcript in embryos. EMBO J 6: 1765-1774.

BODENSTEIN, D. (1950). The postembryonic development of Drosophila. In Biology of Drosophila. (ed. Demerec, M.) pp.275-367. Wiley, New York.

BRÖNNER, G. and JACKLE, H. (1991). Control and function of terminal gap gene activity in the posterior pole region of the Drosophila embryo. Mech Dev35:205-211.

CAMPOS-ORTEGA, J.A. and HARTENSTEIN, V. (1985). In The Embryonic Development of Drosophila Melanogaster, Springer-Verlag, Berlin.

CHEN, C.K., KUHNLEIN, R.P., EULENBERG, K.G., VINCENT, S., AFFOLTER, M. and SCHUH, R. (1998). The transcription factor KNIRPS and KNIRPS RELATED control cell migration and branch morphogenesis during Drosophila tracheal development. Development 125: 4959-4968.

FUSS, B. and $\mathrm{HOCH}$, M. (2002). Notch signaling control cell fate specification along the dorsoventral axis of the Drosophila gut. Curr Biol 12: 171-179.

FUSS, B., MEISSNER, T., BAUER, R., LEHMANN, C., ECKARDT, F. and $\mathrm{HOCH}, \mathrm{M}$. (2001). Control of endoreduplication domains in the Drosophila gut by the knirps and knirps-related genes. Mech Dev 100: 15-23.

GREEN, R. B., HATINI, V., JOHANSEN, K.A., LIU, X. and LENGYEL, J.A. (2002). Drumstick is a zinc finger protein that antagonizes Lines to control patterning 
and morphogenesis of the Drosophila hindgut. Development 129: 3645-3656.

GREENWOOD, S. and STRUHL, G. (1997). Different levels of Ras activity can specify distinct transcriptional and morphological consequences in early Drosophila embryos. Development 124: 4879-4886.

HARBECKE, R. and JANNING, W. (1989). The segmentation gene Krüppel of Drosophila Melanogaster has homeotic properties. Genes Dev 3: 114-122.

HARRISON, D.A., MCCOON, P.E., BINARI, R., GILMAN, M. and PERRIMON, N. (1998). Drosophila unpaired encodes a secreted protein that activates the JAK signaling pathway. Genes Dev 12: 3252-3263.

HITANI, V., GREEN, R.B., LENGYEL, J. A., BARY, S. J. and DINARDO, S. (2005). The Drumstick/Lines/Bowl regulatory pathway links antagonistic Hedgehog and Wingless signaling inputs to epidermal cell differentiation. Genes Dev19:709-718.

HAYES, S.A., MILLER, J.M. and HOSHIZAKI, D.K. (2001). serpent, a GATA-like transcription factor gene, induces fat-cell development in Drosophila melanogaster. Development 128: 1193-1200.

$\mathrm{HOCH}, \mathrm{M}$. and PANKRATZ, M.J. (1996). Control of gut development by forkhead and signaling molecules in Drosophila. Mech Dev 58: 3-14.

IWAKI, D.D., JOHANSEN, K.A., SINGER, J.B. and LENGYEL, J.A. (2001). drumstick, bowl, and lines are required for patterning and cell rearrangement in the Drosophila embryonic hindgut. Dev Biol 240: 611-626.

JOHANSEN K.A., GREEN, R.B., IWAKI, D.D., HERNANDEZ, J.B. and LENGYEL, J.A. (2003). The Drm-Bowl-Lin relief-of-repression hierarchy controls fore- and hindgut patterning and morphogenesis. Mech Dev 120: 1139-1151.

KISPERT, A., HERRMANN, B.G., LEPTIN, M. and REUTER, R. (1994). Homologs of the mouse Brachyury gene are involved in the specification of posterior terminal structures in Drosophila, Tribolium, and Locusta. Genes Dev 8: 2137-2150.

LENGYEL, J.A. and IWAKI, D.D. (2002). It takes guts: the Drosophila hindgut as a model system for organogenesis. Dev Biol 243: 1-19.

LENGYEL, J.A. and LIU, X.J. (1998). Posterior gut development in Drosophila: A model system for identifying genes controlling epithelial morphogenesis. Cell Res 8: 273-284.

LIU, S. and JACK, J. (1992). Regulatory interactions and role in cell type specification of the Malpighian tubules by the cut, Krüppel, and caudal genes of Drosophila. Dev Biol 150: 133-143.

MARTIN, J. R., RAIBAUD, A. and OLLO, R. (1994). Terminal pattern elements in Drosophila embryo induced by the torso-like protein. Nature 367: 741-745.

MURAKAMI, R. and SHIOTSUKI, Y. (2001). Ultrastructure of the hindgut of Drosophila larva, with spacial reference to the domains identified by specefic genes expression pattern. J Morphol 248: 144-150.

MURAKAMI, R., OKUMURA, T. and UCHIYAMA, H. (2005). GATA factors as key regulatory molecules in the development of Drosophila endoderm. Develop Growth Differ 47: 581-589.

MURAKAMI, R., SHIGENAGA, A., KAWAKITA, M., TAKIMOTO, K., YAMAOKA, I., AKASAKA, K. and SHIMADA, H. (1995). aproctous, a locus that is necessary for the development of the proctodeum in Drosophila embryos, encodes a homolog of the vertebrate Brachyury gene. Roux's Arch Dev Biol 205: 89-96.

MURAKAMI, R., SHIGENAGA, A., KAWANO, E., MATSUMOTO, A., YAMAOKA, I. and TANIMURA, T. (1994). Novel tissue units of regional differentiation in the gut epithelium of Drosophila, as revealed by P-element-mediated detection of enhancer. Roux's Arch Dev Biol 203: 243-249.
MURAKAMI, R., TAKASHIMA. S. and HAMAGUCHI, T. (1999). Developmental genetics of the Drosophila gut: specification of primordial, subdivision and overtdifferentiation. Cell Mol Biol 45: 661-676.

NUSSLEIN-VOLHARD, C., FROHNHOFER, H. and LEHMANN, R. (1987). Determination of anteroposterior polarity in Drosophila. Science 238: 1675-1681.

OKUMURA, T., MATSUMOTO, A., TANIMURA, T. and MURAKAMI, R. (2005). An endoderm-specific GATA factor gene, dGATAe, is required for the terminal differentiation of the Drosophila endoderm. Dev Biol 278: 576-586.

PANKRATZ, M.J. and $\mathrm{HOCH}$, M. (1995). Control of epithelial morphogenesis by cell signaling and integrin molecules in the Drosophila foregut. Development 121: 1885-1898.

PIGNONI, F., BALDARELLI, R. M., STEINGRIMSSON, E., DIAZ, R. J., PATAPOUTIAN, A., MERRIAM, J. R., and LENGYEL, J. A. (1990). The Drosophila gene tailless is expressed at the embryonic termini and is a member of the steroid receptor super family. Cell 62: 151-163.

REHORN, K.P., THELEN, H., MICHELSON, A.M. and REUTER, R. (1996). Amolecular aspect of hematopoiesis and endoderm development common to vertebrates and Drosophila. Development 122: 4023-4031.

REDEMANN, N., GAUL, U. and JACKLE, H. (1988). Disruption of a putative Cyszinc interaction eliminates the biological activity of the Krüppel finger protein. Nature 332: 90-92.

REUTER, R. (1994). The gene serpent has homeotic properties and specifies endoderm versus ectoderm within the Drosophila gut. Development 120: 1123-1135.

REUTER, R. and LEPTIN, M. (1994). Interacting function of snail, twist and huckebein during the early development of germ layer in Drosophila. Development 120: 1137-1150.

SAVANT-BHONSALE, S. and MONTELL, D. J. (1993). torso-like encodes the localized determinant of Drosophila terminal pattern formation. Genes Dev 7: 2548-2555.

SIMEONE A., ROSARIA D APICE, M., NIGRE, V., CASANOVA, J., GRAZIANI, F., ACAMPORA, D. and AVANTAGGIATO, V. (1994). orthopedia, a Novel Homeobox containing gene expressed in developing CNS of both Mouse and Drosophila. Neuron 13: 83-101.

SINGER, J. B., HARBECKE, R., KUSCH, T., REUTER, R. and LENGYEL, J. A. (1996). Drosophila brachyenteron regulates gene activity and morphogenesis in the gut. Development 122: 3707-3718.

SKAER, H. (1993). The Alimentary Canal. In: Developmental Biology of Drosophila melamogester (ed. Bate, M. and Martinez Arias, A.) pp.941-1012. Plainview, NY Cold Spring Harbor Laboratory Press.

SPRENGER, F. and NUSSLEIN-VOLHARD, C. (1992). Torso receptor activity is regulated by a diffusible ligand produced at the extracellular terminal regions of the Drosophila egg. Cell 71: 987-1001.

TAKASHIMA, S. and MURAKAMI, R. (2001). Regulation of pattern formation in the Drosophila hindgut by wg, hh, dpp, and en. Mech Dev 101: 79-90.

TAKASHIMA, S., YOSHIMORI, H., YAMASAKI, N., MATSUNO, K. and MURAKAMI, R. (2002). Cell fate choice and boundary formation by combined action of Notch and engrailed in the Drosophila hindgut. Dev Genes Evol 212: 534-541.

TAUTZ, D. and PFEIFLE, C. (1989). A non-radioactive in situ hybridization method for the localization of specific RNAs in Drosophila embryos reveals translational control of the segmentation gene hunchback. Chromosoma 98: 81-85. 


\section{Further Related Reading, published previously in the Int. J. Dev. Biol.}

Hedgehog signalling is required for cloacal development in the zebrafish embryo Caroline A. Parkin, Claire E. Allen and Philip W. Ingham* Int. J. Dev. Biol. 53: 45 - 57 (2009)

Cdx2 specifies the differentiation of morphological as well as functional absorptive enterocytes of the small intestine Hiroyuki Mutoh, Kiichi Satoh, Hiroto Kita, Hirotsugu Sakamoto, Hiroko Hayakawa, Hironori Yamamoto, Norio Isoda, Kiichi Tamada, Kenichi Ido and Kentaro Sugano

Int. J. Dev. Biol. (2005) 49: 867-871

Molecular analysis of the determination of developmental fate in the small intestinal epithelium in the chicken embryo Hiroaki Hiramatsu and Sadao Yasugi

Int. J. Dev. Biol. (2004) 48: 1141 - 1148

Analysis of mouse eye development with chimeras and mosaics J. Martin Collinson, Robert E. Hill and John D. West Int. J. Dev. Biol. (2004) 48: 793-804

Terminal differentiation of palatal medial edge epithelial cells in vitro is not necessarily dependent on palatal shelf contact and midline epithelial seam formation

Toshiya Takigawa and Kohei Shiota Int. J. Dev. Biol. (2004) 48: 307-317

Physiological and induced apoptosis in sea urchin larvae undergoing metamorphosis.

Maria C Roccheri, Cinzia Tipa, Rosa Bonaventura and Valeria Matranga

Int. J. Dev. Biol. (2002) 46: 801-806

mgm 1, the earliest sex-specific germline marker in Drosophila, reflects expression of the gene esg in male stem cells. Adrian Streit, Luca Bernasconi, Pavel Sergeev, Alex Cruz and Monica Steinmann-Zwicky Int. J. Dev. Biol. (2002) 46: 159-166

Early establishment of epithelial apoptosis in the developing human small intestine.

P H Vachon, E Cardin, C Harnois, J C Reed and A Vézina

Int. J. Dev. Biol. (2000) 44: 891-898

Intestinal fatty acid binding protein gene expression reveals the cephalocaudal patterning during zebrafish gut morphogenesis. M André, S Ando, C Ballagny, M Durliat, G Poupard, C Briançon and P J Babin

Int. J. Dev. Biol. (2000) 44: 249-252

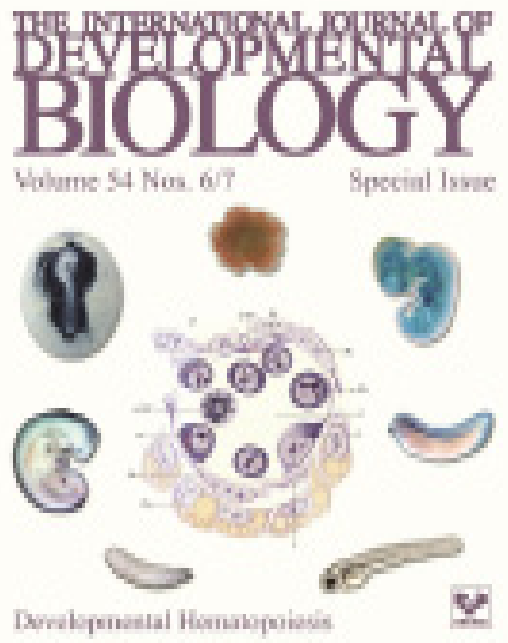

5 yr ISI Impact Factor $(2010)=2.961$

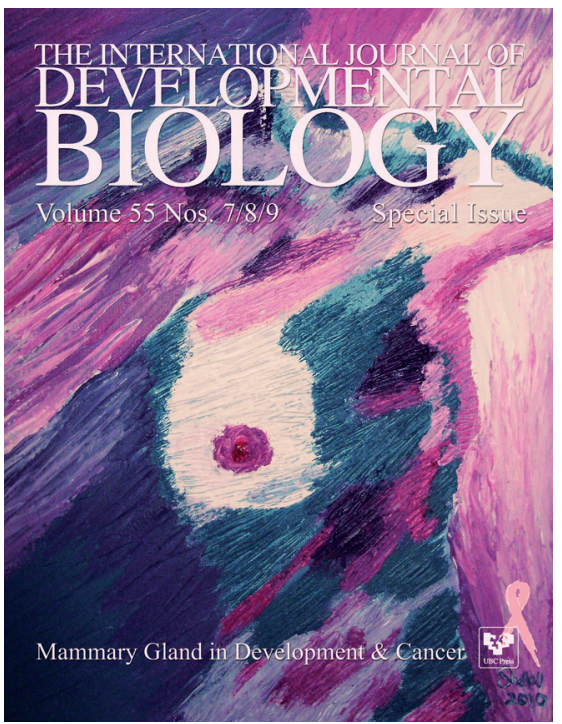

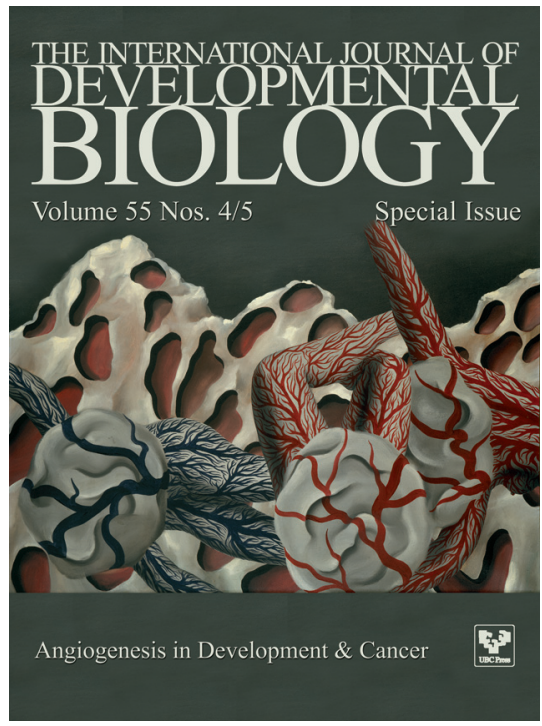

